\title{
Some properties of Chebyshev polynomials
}

\section{Seon-Hong Kim ${ }^{*}$}

\section{"Correspondence:}

shkim17@sookmyung.ac.kr

Department of Mathematics,

Sookmyung Women's University,

Seoul, 140-742, Korea

\begin{abstract}
In this paper we obtain some new bounds for Chebyshev polynomials and their analogues. They lead to the results about zero distributions of certain sums of Chebyshev polynomials and their analogues. Also we get an interesting property about the integrals of certain sums of Chebyshev polynomials.
\end{abstract}

Keywords: Chebyshev polynomials; bounds; sums; zeros

\section{Introduction}

Let $T_{n}(x)$ and $U_{n}(x)$ be the Chebyshev polynomials of the first kind and of the second kind, respectively. These polynomials satisfy the recurrence relations

$$
\begin{array}{ll}
T_{0}(x)=1, & T_{1}(x)=x, \quad T_{n+1}(x)=2 x T_{n}(x)-T_{n-1}(x) \quad(n \geq 1) \\
U_{0}(x)=1, & U_{1}(x)=2 x, \quad U_{n+1}(x)=2 x U_{n}(x)-U_{n-1}(x) \quad(n \geq 1) .
\end{array}
$$

Chebyshev polynomials are of great importance in many areas of mathematics, particularly approximation theory. Many papers and books [3, 4] have been written about these polynomials. Chebyshev polynomials defined on $[-1,1]$ are well understood, but the polynomials of complex arguments are less so. Reported here are several bounds for Chebyshev polynomials defined on $\mathbb{C}$ including zero distributions of certain sums of Chebyshev polynomials. Moreover, we will introduce certain analogues of Chebyshev polynomials and study their properties. Also we get an interesting property about the integrals of certain sums of Chebyshev polynomials.

Other generalized Chebyshev polynomials (known as Shabat polynomials) have been introduced in [5] and they are studied in the theory of graphs on surfaces and curves over number fields. For a survey in this area, see [6].

For $n \geq 1$ and $2-\epsilon>1+\epsilon \geq 1$, i.e., $0 \leq \epsilon<1 / 2$, we let

$$
\begin{aligned}
& T_{n, \epsilon}(z):=(1+\epsilon)\left(T_{n}(z)-T_{n}(0)\right)+T_{n}(0), \\
& U_{n, \epsilon}(z):=(2-\epsilon)\left(U_{n}(z)-U_{n}(0)\right)+U_{n}(0) .
\end{aligned}
$$

In detail,

$$
\begin{aligned}
& T_{n}(0)=U_{n}(0)= \begin{cases}0 & \text { if } n \text { is odd, } \\
(-1)^{n / 2} & \text { if } n \text { is even, }\end{cases} \\
& T_{0, \epsilon}(z)=U_{0, \epsilon}(z)=T_{0}(z)=U_{0}(z)=1,
\end{aligned}
$$


and it is easy to show that for an odd integer $n$,

$$
\begin{aligned}
& T_{n+1, \epsilon}(z)=2 z T_{n, \epsilon}(z)-T_{n-1, \epsilon}(z), \\
& U_{n+1, \epsilon}(z)=2 z U_{n, \epsilon}(z)-U_{n-1, \epsilon}(z) .
\end{aligned}
$$

Since $T_{n, 0}(z)=T_{n}(z)$, we may apply results about $T_{n, \epsilon}(z)$ to those about $T_{n}(z)$. But $U_{n, 1}(z)=$ $U_{n}(z)$ and $\epsilon$ was a nonnegative real number less than $1 / 2$, and so properties of $U_{n}(z)$ will be investigated separately from those of $U_{n, \epsilon}(z)$.

\section{New results}

In this section we list some new results related to the bounds of Chebyshev polynomials $T_{n}(z), U_{n}(z)$ and their analogues $T_{n, \epsilon}(z), U_{n, \epsilon}(z)$ defined on $\mathbb{C}$ including zero distributions of certain sums of Chebyshev polynomials and their analogues. And we will get an interesting property about the integrals of certain sums of Chebyshev polynomials. We first begin with properties about bounds of $T_{n}(z), U_{n}(z), T_{n, \epsilon}(z)$ and $U_{n, \epsilon}(z)$. We may compute that for $n \geq 1$,

$$
T_{n, \epsilon}(z)= \begin{cases}(1+\epsilon) T_{n}(z) & \text { if } n \text { is odd, } \\ (1+\epsilon) T_{n}(z)-\epsilon & \text { if } n \text { is even and } 4 \mid n, \\ (1+\epsilon) T_{n}(z)+\epsilon & \text { if } n \text { is even and } 4 \nmid n,\end{cases}
$$

and

$$
U_{n, \epsilon}(z)= \begin{cases}(2-\epsilon) U_{n}(z) & \text { if } n \text { is odd, } \\ (2-\epsilon) U_{n}(z)-1+\epsilon & \text { if } n \text { is even and } 4 \mid n \\ (2-\epsilon) U_{n}(z)+1-\epsilon & \text { if } n \text { is even and } 4 \nmid n\end{cases}
$$

Proposition 1 Suppose that $z$ is a complex number satisfying $|z| \geq 1$. Then for $n \geq 1$,

$$
\left|U_{n}(z)\right|-\left|U_{n-1}(z)\right| \geq 1
$$

and

$$
\left|U_{n}(z)\right|-\left|T_{n}(z)\right| \geq 1
$$

Also

$$
\left|U_{n, \epsilon}(z)\right|-\left|U_{n-1, \epsilon}(z)\right| \geq 1
$$

and

$$
\left|U_{n, \epsilon}(z)\right|-\left|T_{n, \epsilon}(z)\right|> \begin{cases}1+\epsilon & \text { if } n \text { is odd } \\ \epsilon & \text { if } n \text { is even. }\end{cases}
$$

Proposition 1 will be used in the proofs of Theorems 4 and 6 . 
Remarks For a complex number $z$ with $|z| \geq 1$, we may follow the procedure of the proof of (2) to obtain

$$
\left|T_{n}(z)\right|-\left|T_{n-1}(z)\right| \geq 0 \quad(n \geq 1)
$$

and

$$
\left|U_{n}(z)\right| \geq n+1 \quad(n \geq 0)
$$

that is best possible since $\left|U_{n}(1)\right|=n+1$. There seem to be larger lower bounds than 1 for $\left|U_{n}(z)\right|-\left|T_{n}(z)\right|$ in (2). First, we observe that

$$
\min _{|z| \geq 1}\left(\left|U_{n}(z)\right|-\left|T_{n}(z)\right|\right) \leq n
$$

because $\left|U_{n}(1)\right|-\left|T_{n}(1)\right|=(n+1)-1=n$. Deciding in some general situations exactly where the minimum occurs seems to be extremely difficult. For example, machine calculation suggests that for $n=4,\left|U_{4}(z)\right|-\left|T_{4}(z)\right|$ takes its minimum $3.91735 \ldots$ in $|z| \geq 1$ at four modulus 1 roots $\pm 0.9953 \ldots \pm i 0.0964 \ldots$ of the polynomial

$$
\begin{aligned}
& 256 x^{16}-5568 x^{14}+47352 x^{12}-176952 x^{10}+268549 x^{8} \\
& -176952 x^{6}+47352 x^{4}-5568 x^{2}+256
\end{aligned}
$$

But we may conjecture that, by numerical computations, the value

$$
\min _{|z| \geq 1}\left(\left|U_{n}(z)\right|-\left|T_{n}(z)\right|\right)
$$

occurs in $|z|=1$ and lies between $n-1 / 2$ and $n$, where $n-1 / 2$ can be replaced by something larger. We now ask naturally what the minimum is for $|z|=t>1$. If one simply looks at the case $z=t$, it seems that $\left|U_{n}(z)\right|-\left|T_{n}(z)\right|$ is close to its minimum at $z=t$. But

$$
\left|U_{n}(t)\right|-\left|T_{n}(t)\right|=U_{n}(t)-T_{n}(t)
$$

is the coefficient of $x^{n-1}$ in the power series expansion of $t /\left(1-2 t x+t^{2}\right)$. In fact,

$$
\begin{aligned}
\frac{t x}{1-2 t x+x^{2}} & =\frac{1}{1-2 t x+x^{2}}-\frac{1-t x}{1-2 t x+x^{2}} \\
& =\sum_{n=0}^{\infty} U_{n}(t) x^{n}-\sum_{n=0}^{\infty} T_{n}(t) x^{n}=\sum_{n=0}^{\infty}\left(U_{n}(t)-T_{n}(t)\right) x^{n} .
\end{aligned}
$$

For $|z| \geq 1,\left|U_{n}(z)\right| \geq\left|T_{n}(z)\right|+1$ by (2). In the following proposition, we obtain an upper bound for arbitrary $z=\cos \theta, \theta \notin \mathbb{R}$.

Proposition 2 Let $z=\cos \theta$, where $\theta=\alpha+i \beta$ and $\beta \neq 0$. Then, for $n \geq 1$,

$$
\left|U_{n}(z)\right| \leq(1+\operatorname{coth} \beta \operatorname{coth} n \beta)\left|T_{n}(z)\right| \text {. }
$$


Remarks With the same notations with Proposition 2, it follows from

$$
|z|^{2}=|\cos \theta|^{2}=\frac{1}{2}(\cos 2 \alpha+\cosh 2 \beta)
$$

that, for $|z|$ large, $|\beta|$ is large. But $\operatorname{coth} \beta \operatorname{coth} n \beta>1$ and

$$
\lim _{\beta \rightarrow \pm \infty} \operatorname{coth} \beta \operatorname{coth} n \beta=1 \text {. }
$$

These imply that the upper bound

$$
(1+\operatorname{coth} \beta \operatorname{coth} n \beta)\left|T_{n}(z)\right|
$$

is greater than $2\left|T_{n}(z)\right|$, but for $|z|$ large, it is close to $2\left|T_{n}(z)\right|$. Also by machine computations (e.g., $n=4$ and $z=100+i 100$ ), we may check that the inequality (5) is sharp.

It is natural to ask about the bounds on the unit circle.

Proposition 3 For $|z|=1$, we have

$$
\frac{1}{n+1}\left|U_{n}(z)\right| \leq\left|T_{n}(z)\right|<\left|U_{n}(z)\right|
$$

Remarks The right inequality in (6) will be shown in Section 3 by using (2). So obtaining a better lower bound than 1 in (2) can improve this inequality. The left inequality in (6) is best possible in the sense that

$$
\frac{1}{n+1}\left|U_{n}( \pm 1)\right|=\left|T_{n}( \pm 1)\right|=1 \text {. }
$$

For $|z|=1$, it is easy to see

$$
\left|T_{n, \epsilon}(z)\right|<\left|U_{n, \epsilon}(z)\right|
$$

by (4), and it seems to be true that

$$
\frac{1}{n+1}\left|U_{n, \epsilon}(z)\right| \leq\left|T_{n, \epsilon}(z)\right|
$$

The proof of (6) will be given in Section 3 by using a well-known identity $U_{n}(z)=T_{n}(z)+$ $z U_{n-1}(z)$. But $U_{n, \epsilon}(z)=T_{n, \epsilon}(z)+z U_{n-1, \epsilon}(z)$ does not hold. So we cannot use this to prove (7) if it is true.

All zeros of the polynomial $T_{n}(z)+U_{n}(z)$ lie in $(-1,1)$. More generally the convex combination of $T_{n}(z)$ and $U_{n}(z)$ has all its zeros in $(-1,1)$. This will be proved in Proposition 5 below. So one might ask: where are the zeros of polynomials like $T_{n}(z)+z^{k} U_{n}(z)$ or $U_{n}(z)+z^{k} T_{n}(z)$ around $|z|=1$ ? The next theorem answers this for $T_{n}(z)+z^{k} U_{n}(z)$.

Theorem 4 Let $P_{1}(z):=T_{n}(z)+z^{k} U_{n}(z)$ for positive integers $n$ and $k$. Then $P_{1}(z)$ has all its zeros in $|z|<1$. Furthermore, for $k$ even, $P_{1}(z)$ has at least $n$ real zeros, and for $k$ odd, $P_{1}(z)$ has at least $n-1$ real zeros. 
Remarks Let $P_{2}(z):=U_{n}(z)+z^{k} T_{n}(z)$ for positive integers $n$ and $k$. We can use the same method as in the proof of Theorem 4 to show that $P_{2}(z)$ has at least $n$ real zeros in $(-1,1)$. Furthermore, for $k$ even, there is no real zero outside $[-1,1]$, and for $k$ odd, there is one more real zero on $z<-1$.

Proposition 5 The polynomial

$$
(1-\lambda) T_{n}(z)+\lambda U_{n}(z) \quad(0 \leq \lambda \leq 1)
$$

has all zeros in $(-1,1)$.

Using analogues of $T_{n}(z)$ and $U_{n}(z)$, we consider analogues of $P_{1}(z)$ and investigate their zero distributions. Define

$$
P_{1, \epsilon}(z):=T_{n, \epsilon}(z)+z^{k} U_{n, \epsilon}(z)
$$

Theorem $6 P_{1, \epsilon}(z)$ has all its zeros in $|z|<1$.

Finally, we get an interesting property about the integrals of sums of Chebyshev polynomials. Observe that

$$
\int_{0}^{2 \pi} T_{n}\left(e^{i \theta}\right) d \theta=\int_{0}^{2 \pi} U_{n}\left(e^{i \theta}\right) d \theta=T_{n}(0) \cdot 2 \pi= \begin{cases}(-1)^{n / 2} & \text { if } n \text { is even } \\ 0 & \text { if } n \text { is odd }\end{cases}
$$

and

$$
\int_{0}^{2 \pi}\left|T_{n}\left(e^{i \theta}\right)\right|^{2} d \theta \quad\left(\text { or } \int_{0}^{2 \pi}\left|U_{n}\left(e^{i \theta}\right)\right|^{2} d \theta\right)
$$

equals

$2 \pi \cdot$ sum of the squares of all coefficients of $T_{n}(z)$ (or $\left.U_{n}(z)\right)$.

For example, from $T_{4}(z)=8 z^{4}-8 z^{2}+1$ and $U_{4}(z)=16 z^{6}-80 z^{4}+24 z^{2}-1$ we can calculate

$$
\begin{aligned}
& \int_{0}^{2 \pi}\left|T_{4}\left(e^{i \theta}\right)\right|^{2} d \theta=2 \pi\left(8^{2}+8^{2}+1\right)=258 \pi \\
& \int_{0}^{2 \pi}\left|U_{4}\left(e^{i \theta}\right)\right|^{2} d \theta=2 \pi\left(16^{2}+12^{2}+1^{2}\right)=802 \pi
\end{aligned}
$$

and we see that these two integrals are different. But for $P_{1}(z)=T_{n}(z)+z^{k} U_{n}(z)$ and $P_{2}(z)=$ $U_{n}(z)+z^{k} T_{n}(z)$, the integrals have the same value.

Proposition 7 For $z=e^{i \theta}$,

$$
\int_{0}^{2 \pi}\left|P_{1}\left(e^{i \theta}\right)\right|^{2} d \theta=\int_{0}^{2 \pi}\left|P_{2}\left(e^{i \theta}\right)\right|^{2} d \theta
$$


Remark It seems to be true that for $k$ large, $z=e^{i \theta}$,

$$
\int_{0}^{2 \pi}\left|P_{1}\left(e^{i \theta}\right)\right| d \theta \approx \int_{0}^{2 \pi}\left|P_{2}\left(e^{i \theta}\right)\right| d \theta
$$

but

$$
\lim _{k \rightarrow \infty} \int_{0}^{2 \pi}\left|P_{1}\left(e^{i \theta}\right)\right| d \theta=\lim _{k \rightarrow \infty} \int_{0}^{2 \pi}\left|P_{2}\left(e^{i \theta}\right)\right| d \theta .
$$

These remain open problems.

\section{Proofs}

Proof of Proposition 1 Suppose that $z$ is a complex number satisfying $|z| \geq 1$. Using (1), for $n \geq 1$, we have

$$
\left|U_{n+1}(z)\right| \geq 2\left|U_{n}(z)\right|-\left|U_{n-1}(z)\right|
$$

and

$$
\left|U_{n+1}(z)\right|-\left|U_{n}(z)\right| \geq\left|U_{n}(z)\right|-\left|U_{n-1}(z)\right| .
$$

Then by recurrence,

$$
\left|U_{n}(z)\right|-\left|U_{n-1}(z)\right| \geq\left|U_{1}(z)\right|-\left|U_{0}(z)\right|=|2 z|-1 \geq 1 .
$$

By (8) and the identity

$$
T_{n}(z)=\frac{1}{2}\left(U_{n}(z)-U_{n-2}(z)\right),
$$

we have

$$
\begin{aligned}
\left|T_{n}(z)\right| & \leq \frac{1}{2}\left|U_{n}(z)\right|+\frac{1}{2}\left|U_{n-2}(z)\right| \\
& \leq \frac{1}{2}\left|U_{n}(z)\right|+\frac{1}{2}\left(\left|U_{n}(z)\right|-2\right) \\
& =\left|U_{n}(z)\right|-1 .
\end{aligned}
$$

Next we prove the results about $U_{n, \epsilon}(z)$ and $T_{n, \epsilon}(z)$. For $n$ odd and $4 \mid n-1$, it follows from the definition of $U_{n, \epsilon}(z)$ and (8) that

$$
\begin{aligned}
\left|U_{n, \epsilon}(z)\right|-\left|U_{n-1, \epsilon}(z)\right| & =\left|(2-\epsilon) U_{n}(z)\right|-\left|(2-\epsilon) U_{n-1}(z)-(1-\epsilon)\right| \\
& \geq\left|(2-\epsilon) U_{n}(z)\right|-\left|(2-\epsilon) U_{n-1}(z)\right|-(1-\epsilon) \\
& =(2-\epsilon)\left(\left|U_{n}(z)\right|-\left|U_{n-1}(z)\right|\right)-(1-\epsilon) \\
& \geq 2-\epsilon-1+\epsilon=1 .
\end{aligned}
$$


This inequality

$$
\left|U_{n, \epsilon}(z)\right|-\left|U_{n-1, \epsilon}(z)\right| \geq 1
$$

for other three cases (i.e., $n$ odd and $4 \nmid n-1, n$ even and $4 \mid n-1, n$ even and $4 \nmid n-1)$ can be proved in the same way. Finally, for $n$ odd, by $2-\epsilon>1+\epsilon$ and (9), we have

$$
\begin{aligned}
\left|U_{n, \epsilon}(z)\right|-\left|T_{n, \epsilon}(z)\right| & =(2-\epsilon)\left|U_{n}(z)\right|-(1+\epsilon)\left|T_{n}(z)\right| \\
& >(1+\epsilon)\left(\left|U_{n}(z)\right|-\left|T_{n}(z)\right|\right) \geq 1+\epsilon .
\end{aligned}
$$

In the same way, we can check that for $n$ even,

$$
\left|U_{n, \epsilon}(z)\right|-\left|T_{n, \epsilon}(z)\right|>\epsilon .
$$

Proof of Proposition 2 Using the identity $U_{n}(z)=T_{n}(z)+z U_{n-1}(z)$, for $z=\cos \theta$ we have

$$
\begin{aligned}
\left|\frac{U_{n}(z)}{T_{n}(z)}\right| & =\left|1+z \frac{U_{n-1}(z)}{T_{n}(z)}\right|=\left|1+\cos \theta \frac{\tan n \theta}{\sin \theta}\right| \\
& =|1+\cot \theta \tan n \theta| \leq 1+|\cot \theta \tan n \theta| .
\end{aligned}
$$

If we set $\theta=\alpha+i \beta, \alpha, \beta \in \mathbb{R}$, then

$$
\begin{aligned}
& \sin \theta=\sin \alpha \cosh \beta+i \cos \alpha \sinh \beta, \\
& \cos \theta=\cos \alpha \cosh \beta-i \sin \alpha \sinh \beta .
\end{aligned}
$$

Since $\bar{z}=\cos \bar{\theta}$, it suffices to consider the case $\beta>0$. The above implies

$$
|\tan \theta|^{2}=\frac{\sin ^{2} \alpha \cos ^{2} \alpha+\sinh ^{2} \beta \cosh ^{2} \beta}{\left(\cos ^{2} \alpha+\sinh ^{2} \beta\right)^{2}} \leq \frac{\sinh ^{2} \beta \cosh ^{2} \beta}{\sinh ^{4} \beta} .
$$

So

$$
|\tan n \theta| \leq \operatorname{coth} n \beta \text {. }
$$

Also

$$
\begin{aligned}
|\sin \theta|^{2} & =\sin ^{2} \alpha \cosh ^{2} \beta+\cos ^{2} \alpha \sinh ^{2} \beta \\
& \geq\left(\sin ^{2} \alpha+\cos ^{2} \alpha\right) \sinh ^{2} \beta
\end{aligned}
$$

and

$$
\begin{aligned}
|\cos \theta|^{2} & =\cos ^{2} \alpha \cosh ^{2} \beta+\sin ^{2} \alpha \sinh ^{2} \beta \\
& \leq\left(\sin ^{2} \alpha+\cos ^{2} \alpha\right) \cosh ^{2} \beta,
\end{aligned}
$$

and so

$$
|\cot \theta| \leq \operatorname{coth} \beta \text {. }
$$


Now we see with (10) and (11) that

$$
|\cot \theta \tan n \theta| \leq \operatorname{coth} \beta \operatorname{coth} n \beta \text {. }
$$

Proof of Proposition 3 Suppose that $z$ is a complex number satisfying $|z|=1$. First, it follows from (2) that

$$
\left|\frac{T_{n}(z)}{U_{n}(z)}\right|<1
$$

Using the identity $U_{n}(z)=T_{n}(z)+z U_{n-1}(z)$, we have

$$
\left|\frac{T_{n}(z)}{U_{n}(z)}\right|=\left|1-z \frac{U_{n-1}(z)}{U_{n}(z)}\right| \geq 1-\left|\frac{U_{n-1}(z)}{U_{n}(z)}\right|
$$

and so it is enough to show that

$$
\left|\frac{U_{n-1}(z)}{U_{n}(z)}\right| \leq 1-\frac{1}{n+1}=\frac{n}{n+1}
$$

We use induction on $n$. For $n=1,\left|\frac{U_{0}(z)}{U_{1}(z)}\right|=\left|\frac{1}{2 z}\right|=\frac{1}{2}$. Assume the result holds for $k$. Then

$$
\left|\frac{U_{k+1}(z)}{U_{k}(z)}\right|=\left|2 z-\frac{U_{k-1}(z)}{U_{k}(z)}\right| \geq 2-\left|\frac{U_{k-1}(z)}{U_{k}(z)}\right| \geq 2-\frac{k}{k+1}=\frac{k+2}{k+1},
$$

and

$$
\left|\frac{U_{k}(z)}{U_{k+1}(z)}\right| \leq \frac{k+1}{k+2}
$$

Proof of Theorem 4 All zeros of $T_{n}(z)$ and $U_{n}(z)$ are real and lie in $(-1,1)$ and for $1 \leq k \leq n$,

$$
T_{n}\left(\cos \frac{(2 k-1) \pi}{2 n}\right)=0, \quad U_{n}\left(\cos \frac{k \pi}{n+1}\right)=0 .
$$

For convenience, by removing 'cos' and the constant $\pi, \cos \frac{(2 k-1) \pi}{2 n}$ and $\cos \frac{k \pi}{n+1}$ can be identified with the ascending chain of rational numbers $\frac{(2 k-1)}{2 n}$ and $\frac{k}{n+1}$, respectively. We may calculate that for $n$ odd

$$
\begin{cases}\frac{2 k-1}{2 n}<\frac{k}{n+1}<\frac{2 k+1}{2 n}, & 1 \leq k<\frac{n+1}{2}, \\ \frac{2 k+1}{2 n}=\frac{k+1}{n+1}, & k=\frac{n-1}{2}, \\ \frac{2 k-1}{2 n}<\frac{k+1}{n+1}<\frac{2 k+1}{2 n}, & \frac{n-1}{2}<k<n-1, \\ \frac{2 k-1}{2 n}<\frac{k+1}{n+1}, & k=n-1\end{cases}
$$

and for $n$ even

$$
\left\{\begin{array}{lll}
\frac{2 k-1}{2 n}<\frac{k}{n+1}<\frac{2 k+1}{2 n}, & & 1 \leq k<\frac{n}{2}, \\
\frac{2 k-1}{2 n}<\frac{k}{n+1}<\frac{k+1}{n+1}<\frac{2 k+1}{2 n}, & & k=\frac{n}{2}, \\
\frac{2 k-1}{2 n}<\frac{k+1}{n+1}<\frac{2 k+1}{2 n}, & & \frac{n}{2}<k<n-1, \\
\frac{2 k-1}{2 n}<\frac{k+1}{n+1}, & & k=n-1 .
\end{array}\right.
$$


By using the above and denoting $\square$ a zero of $T_{n}(z), \square$ a zero of $z^{k} U_{n}(z)$, we see that, for $n$ even, all zeros between -1 and 1 listed in increasing order are of the form

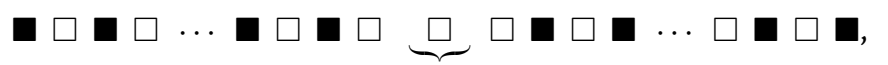

where the center $\underbrace{\square}$ is the 0 that is the zero of $z^{k}$. For $n$ odd, all zeros listed in increasing order are of the form

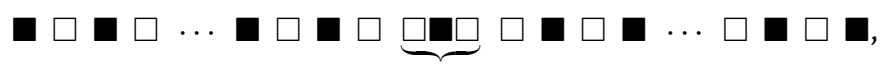

where the center $\underbrace{\square} \square$ means that all those three numbers $\square, \square, \square$ are $\cos \frac{\pi}{2}=0$ and one $\square$ comes from the zero of $z^{k}$. Now consider sign changes using the above two chains in increasing order so that for $n$ odd or even we may check that, if $k$ is even, $P_{1}(z)$ has at least $n$ real zeros in $(-1,1)$, and if $k$ is odd, $P_{1}(z)$ has at least $n-1$ real zeros in $(-1,1)$. On the other hand, the zeros $z$ of $P_{1}(z)$ satisfy

$$
\left|\frac{T_{n}(z)}{U_{n}(z)}\right|=|z|^{k} .
$$

If $|z| \geq 1$, then $\left|T_{n}(z)\right| \geq\left|U_{n}(z)\right|$, which contradicts (2). Thus all zeros of $P_{1}(z)$ lie in $|z|<1$.

'Bad pairs' of polynomial zeros were defined in [2]. It is an easy consequence of Fell [1] that, if the all zeros of $T_{n}(z)$ and $U_{n}(z)$ form 'good pairs', their convex combination has all its zeros real.

Proof of Proposition 5 Following the proof of Theorem 4, we may see that for $n$ even, all zeros $T_{n}(z)$ and $U_{n}(z)$ between -1 and 1 listed in increasing order are of the form

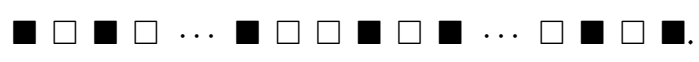

For $n$ odd, all zeros listed in increasing order are of the form

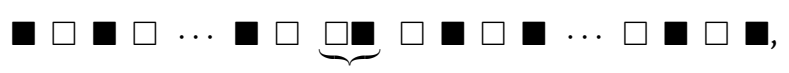

where the center $\underbrace{\square}$ means that both numbers $\square, \mathbf{a}$ are $\cos \frac{\pi}{2}=0$. Thus we can see that for $n$ even, all zeros of $T_{n}(z)$ and $U_{n}(z)$ form good pairs, and for $n$ odd, all pairs from integral polynomials $T_{n}(z) / z$ and $U_{n}(z) / z$ are good. It follows that, by Fell [1], all zeros of the convex combination are real and in $(-1,1)$.

Proof of Theorem 6 The zeros $z$ of $P_{1, \epsilon}(z)$ satisfy

$$
\left|\frac{T_{n, \epsilon}(z)}{U_{n, \epsilon}(z)}\right|=|z|^{k} .
$$

If $|z| \geq 1$, then $\left|T_{n, \epsilon}(z)\right| \geq\left|U_{n, \epsilon}(z)\right|$, which contradicts (4).

Proof of Proposition 7 Using $|z|^{2}=z \bar{z}$, we have

$$
\left|P_{1}(z)\right|^{2}=\left(T_{n}\left(e^{i \theta}\right)+e^{i k \theta} U_{n}\left(e^{i \theta}\right)\right)\left(T_{n}\left(e^{-i \theta}\right)+e^{-i k \theta} U_{n}\left(e^{-i \theta}\right)\right)
$$


and

$$
\left|P_{2}(z)\right|^{2}=\left(U_{n}\left(e^{i \theta}\right)+e^{i k \theta} T_{n}\left(e^{i \theta}\right)\right)\left(U_{n}\left(e^{-i \theta}\right)+e^{-i k \theta} T_{n}\left(e^{-i \theta}\right)\right) .
$$

So we only need to show that

$$
\begin{aligned}
& \int_{0}^{2 \pi}\left(e^{-i k \theta} T_{n}\left(e^{i \theta}\right) U_{n}\left(e^{-i \theta}\right)+e^{i k \theta} T_{n}\left(e^{-i \theta}\right) U_{n}\left(e^{i \theta}\right)\right) d \theta \\
& =\int_{0}^{2 \pi}\left(e^{-i k \theta} T_{n}\left(e^{-i \theta}\right) U_{n}\left(e^{i \theta}\right)+e^{i k \theta} T_{n}\left(e^{i \theta}\right) U_{n}\left(e^{-i \theta}\right)\right) d \theta
\end{aligned}
$$

and

$$
\begin{gathered}
\int_{0}^{2 \pi}\left(-\sin k \theta T_{n}\left(e^{i \theta}\right) U_{n}\left(e^{-i \theta}\right)+\sin k \theta T_{n}\left(e^{-i \theta}\right) U_{n}\left(e^{i \theta}\right)\right) d \theta \\
\quad=\int_{0}^{2 \pi}\left(-\sin k \theta T_{n}\left(e^{-i \theta}\right) U_{n}\left(e^{i \theta}\right)+\sin k \theta T_{n}\left(e^{i \theta}\right) U_{n}\left(e^{-i \theta}\right)\right) d \theta .
\end{gathered}
$$

But this equality follows from just replacing the variable $\theta$ by $-\theta$.

\section{Competing interests}

The author declares that they have no competing interests.

\section{Acknowledgements}

The author wishes to thank Professor Kenneth B. Stolarsky who let the author know some questions in this paper. The author is grateful to the referee of this paper for useful comments and suggestions that led to further development of an earlier version. This research was supported by Basic Science Research Program through the National Research Foundation of Korea (NRF) funded by the Ministry of Education, Science and Technology (2010-0011010).

Received: 26 October 2011 Accepted: 16 July 2012 Published: 31 July 2012

\section{References}

1. Fell, HJ: On the zeros of convex combinations of polynomials. Pac. J. Math. 89, 43-50 (1980)

2. Kim, S-H: Bad pairs of polynomial zeros. Commun. Korean Math. Soc. 15, 697-706 (2000)

3. Mason, JC, Handscomb, DC: Chebyshev Polynomials. Chapman \& Hall/CRC, Boca Raton (2003)

4. Rivlin, TJ: Chebyshev Polynomials. From Approximation Theory to Algebra and Number Theory. Pure and Applied Mathematics. Wiley, New York (1990)

5. Shabat, GB, Voevodsky, VA: Drawing curves over number fields. In: The Grothendieck Festschrift, vol. 3, pp. $199-227$. Birkhäuser, Basel (1990)

6. Shabat, GB, Zvonkin, A: Plane trees and algebraic numbers, Jerusalem combinatorics '93. Contemp. Math., vol. 178, pp. 233-275. Am. Math. Soc., Providence (1994)

doi:10.1186/1029-242X-2012-167

Cite this article as: Kim: Some properties of Chebyshev polynomials. Journal of Inequalities and Applications 2012 2012:167. 